
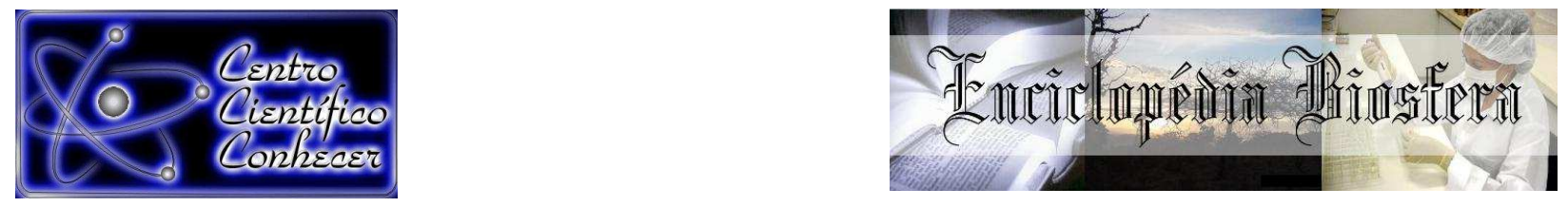

\title{
ANÁLISE DAS EMISSÕES ATMOSFÉRICAS, RENDIMENTO E NÍVEL DE RUÍDO EM ÔNIBUS MOVIDO A BIOMETANO - UM ESTUDO DE CASO
}

\author{
Marildo Guerini Filho ${ }^{1}$, Luiza Marques de Siqueira ${ }^{2}$, Roger Coroto $^{3}$, Odorico Konrad ${ }^{4}$ \\ ${ }^{1}$ Engenheiro Ambiental. Mestrando em Sensoriamento Remoto pela Universidade \\ Federal do Rio Grande do Sul (UFRGS) (mfilho1@universo.univates.br). \\ ${ }^{2}$ Engenheira Ambiental pela Universidade do Vale do Taquari (Univates). \\ ${ }^{3}$ Engenheiro Mecânico. Mestrando em System Engineering pela Universitätdes \\ Saarlandes. \\ ${ }^{4}$ Professor Doutor em Engenharia Ambiental e Sanitária pela Montanuniversitat \\ Leoben.
}

Recebido em: 02/10/2017 - Aprovado em: 21/11/2017 - Publicado em: 05/12/2017 DOI: 10.18677/EnciBio_2017B113

\begin{abstract}
Muito se fala em sustentabilidade, porém, muitos não sabem a importância deste conceito que significa aliar o desenvolvimento sem esgotar os recursos para as gerações futuras. Neste contexto o biometano é um combustível gasoso de origem renovável, produzido a partir da decomposição de substratos orgânicos por meio da biodigestão anaeróbia, possuindo características similares ao Gás natural veicular (GNV). Este estudo apresenta uma avaliação das emissões atmosféricas, do consumo de combustível (rendimento) e do nível de ruído gerado por ônibus movido a biometano no estado do Rio Grande do Sul. As análises foram realizadas através de três equipamentos, Chemist $404 \mathrm{~S}$ que analisou os parâmetros: $\mathrm{O}_{2}, \mathrm{CO}_{2}, \mathrm{NO}, \mathrm{SO}_{2}$, NOx, Temp. gás e Temp. ar, Discovery $\mathrm{G} 4$ avaliou: $\mathrm{CO}, \mathrm{CO}_{\text {corrigido, }} \mathrm{HC}, \mathrm{HC}_{\text {corrigido., }}$ um Decibilímetro DEC 490 para avaliar o nível de ruído e os testes de rendimento foram realizados com o veículo em movimento. Os resultados obtidos de emissões de poluentes e ruídos atmosféricos ficaram significativamente abaixo dos níveis permitidos na legislação. Os achados demonstram a potencialidade e as características específicas do combustível gasoso, evidenciando a possibilidade da substituição parcial ou total dos combustíveis fósseis pelo biometano, e paralelamente pode representar a geração de novos postos de emprego e renda para as atividades agrícolas e industriais, a partir da produção em larga escala.
\end{abstract}

RESUMO

PALAVRAS-CHAVE: emissões veiculares, energia renovável, poluição atmosférica.

\section{ANALYSIS OF ATMOSPHERIC EMISSIONS, PERFORMANCE AND NOISE LEVEL IN BUS MOVED TO BIOMETANO}

\begin{abstract}
So much is said about sustainability, however many do not know the importance of this concept that in a few words means the development does not end resources for future generations. In this context biomethane is a gaseous fuel from renewable sources, produced from the decomposition of organic substrates by means of
\end{abstract}


anaerobic digestion, having characteristics similar to compressed natural gas (CNG). This paper presents an assessment of atmospheric emissions, fuel consumption (income) and noise level generated by a bus powered by biomethane, held in Univates in Lajeado - RS. Analyses were performed by three equipment Chemist 404S which analyzed parameters; $\mathrm{O}_{2}, \mathrm{CO}_{2}, \mathrm{NO}, \mathrm{SO}_{2}, \mathrm{NOx}$, Temp. Gas and Temp. Air, Discovery G4 evaluated: CO, COcorrected, HC, HCcorrected, an Decibilímetro DEC 490 to assess the noise level and performance tests were carried out with the vehicle in motion. The results of pollutant emissions and atmospheric noise were significantly below the levels allowed by law. The findings demonstrate the potential and specific characteristics of gaseous fuel, demonstrating the possibility of partial or total replacement of fossil fuels by biomethane, and at the same time may represent the generation of new jobs and income for the agricultural and industrial activities, from large-scale production.

KEYWORDS: vehicle emissions, renewable energy, air pollution.

\section{INTRODUÇÃO}

A constante preocupação com o meio ambiente e a necessidade de reduzir o consumo dos combustíveis fósseis, vem aumentando os incentivos e a participação das energias renováveis na matriz energética nacional e mundial. A crescente utilização dos combustíveis fósseis, desde a era da revolução industrial, já causou o aumento de aproximadamente $30 \%$ dos chamados greenhause gases ou denominados gases de efeito estufa. Estes gases são considerados por Vinoth Kumar e Kasturi Bai (2008) e Bilotta e Ross (2016), como os principais causadores do aquecimento global e presume-se que estão diretamente ligados as recorrentes mudanças climáticas em todo o mundo (JOHNSON; HELTZEL, 2016).

O lançamento descontrolado de materiais poluentes na atmosfera é uma das conseqüências da deficiência de políticas públicas que regulamentam as emissões atmosféricas de indústrias e veículos automotores. Neste contexto, diversas pesquisas científicas relacionam doenças como o câncer, 0 acidente vascular cerebral, os problemas respiratórios e cardíacos a estes poluentes (FREITAS et al., 2004; GONÇALVES et al., 2005; BRASIL, 2011;).

O transporte através de veículos pesados geralmente está associado à produção de algum tipo de poluição, seja sonora, no que se refere aos ruídos gerados pelos veículos, seja atmosférica, a qual está associada diretamente com as partículas poluentes que são emitidas pelos escapamentos dos veículos ou a intrusão visual que está relacionada com a degradação da paisagem urbana (DRUMM et al., 2014).

Neste contexto, as energias renováveis de ciclo curto se apresentam como excelentes opções para minimizar os impactos sociais e ambientais gerados pela incessante utilização dos combustíveis fósseis. Dentre os diferentes meios de produção de energias renováveis pode-se citar a valorização energética de resíduos orgânicos por meio da biodigestão anaeróbica, esta apresenta três grandes benefícios: o primeiro é a geração de uma nova fonte de energia limpa (biogás), o segundo a redução dos impactos sobre a atmosfera em função da redução de emissões de gases altamente poluentes e a diminuição da poluição do solo e águas (subterrâneas e superficiais) visto que, o resíduo orgânico ao passar pelo processo estará em condições de biofertilizante, minimizando os danos ao solo e águas se utilizado em proporções adequadas (STARR et al., 2012; COIMBRA-ARAÚJO et al., 2014; KARLSSON et al., 2014). 
O biogás é um biocombustível de fonte renovável de ciclo curto, constituído principalmente por dois componentes: metano $(55-65 \%)$ e dióxido de carbono (30 - 35\%), com uma pequena parcela de outros gases (CÁCERES et al., 2012; LUMI; KONRAD; MARDER, 2015). Após processos de purificação o biogás tem como produto final o biometano, com valores acima de $96 \% \mathrm{CH}_{4}$, apresentando características semelhantes ao gás natural veicular.

$\mathrm{Na}$ Europa a EBA (Europian Biogas Association) é responsável pelo gerenciamento da produção de biogás. Atualmente estima-se que a produção total de biogás na Europa seja de 14 bilhões $\mathrm{m}^{3}$ com perspectivas de duplicar está produção até 2020 (EBA, 2011). No Brasil, a comercialização do biometano como biocombustível segue as especificações técnicas estabelecidas pelas Resoluções da ANP no 8 de 30/01/2015 e no 685 de 29/06/2017 e pela Lei oㅜ 14.864/2016 no Estado do Rio Grande do Sul (EPE, 2017).

Conforme o exposto acima, esta pesquisa teve como objetivo avaliar as potencialidades da utilização do biometano como combustível gasoso em um ônibus, através de testes de emissões de gases atmosféricos, rendimento e nível de ruído no Estado do Rio Grande do Sul.

\section{MATERIAL E METODOS}

A execução deste estudo foi realizada utilizando um veículo ônibus, com peso bruto de $24.600 \mathrm{~kg}$ e aproximadamente 15 metros de comprimento. O motor do veículo é OC09 102/250 kW, 5 cilindros, placa especial BFY-9657 (Figura 1).

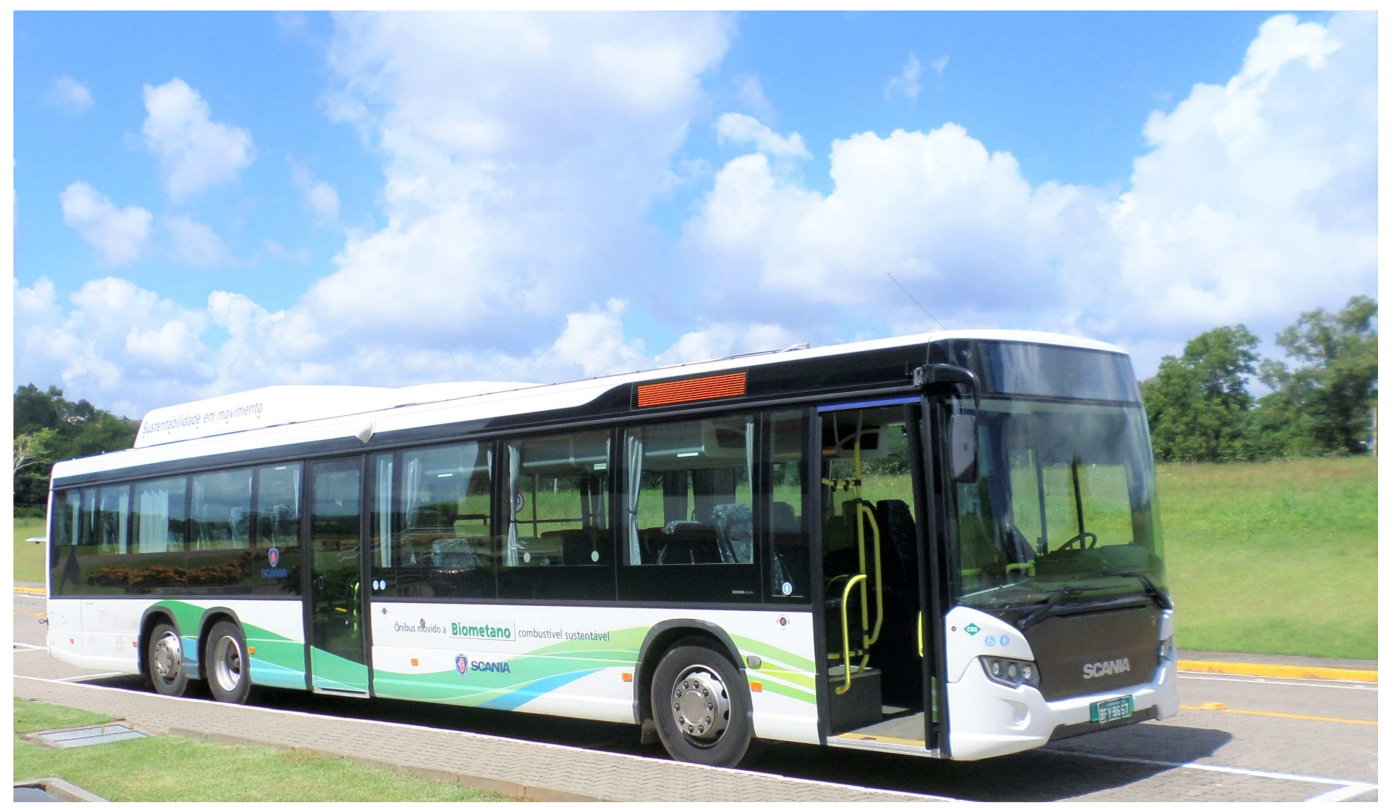

FIGURA 1: ônibus utilizado nos testes. Fonte: autores (2017)

Para determinar as emissões de gases atmosféricos foram utilizados dois analisadores de gases: Chemist 404S e Discovery G4. O analisador de gases Chemist 404S consiste em um dispositivo portátil com uma sonda que é acoplada no escapamento do veículo para análise dos seguintes parâmetros: $\mathrm{O}_{2}, \mathrm{CO}_{2}, \mathrm{NO}$, $\mathrm{SO}_{2}, \mathrm{NOx}$, Temp. gás e Temp. ar.

$\mathrm{O}$ analisador de gases Discovery G4 consiste em um equipamento conectado a um computador que após o início do teste apresenta os resultados através de um 
software específico. Os resultados apresentados ao final de cada teste indicam a aprovação ou reprovação do veículo por meio do confronto dos parâmetros obtidos com os especificados em legislação. Com o equipamento pode-se analisar $\mathrm{CO}$, $\mathrm{CO}_{\text {corrigido, }} \mathrm{HC}, \mathrm{HC}_{\text {corrigido }}$.

Todos os ensaios foram realizados em triplicatas com o veículo estático em duas diferentes rotações do motor: $600 \mathrm{rpm}$ e $2500 \mathrm{rpm}$. O teste de rendimento foi realizado durante 30 dias, em que o itinerário do veículo passava em quatro municípios no estado do Rio Grande do Sul.

Com relação aos fatores de emissões de poluentes atmosféricos veiculares, foram utilizados os limiares conforme a Resolução no 418/2009 (BRASIL, 2009) do Conselho Nacional do Meio Ambiente (CONAMA) para os parâmetros de CO e HC. Esta normativa dispõe sobre critérios para a elaboração de Planos de Controle de Poluição Veicular - PCPV e para a implantação de Programas de Inspeção e Manutenção de Veículos em Uso. Para a realização das avaliações da geração de ruídos, foi utilizado um decibilímetro de ruído da marca INSTRUTHERM modelo DEC 490 e computador para organização dos dados obtidos nas medições.

\section{RESULTADOS E DISCUSSÕES}

Neste capítulo serão apresentados os resultados obtidos e discutidos em relação ao atual estado da arte, bem como comparado com a legislação vigente. $\mathrm{Na}$ Tabela 1 estão apresentadas as médias obtidas nos testes de emissões atmosféricas nas duas rotações avaliadas.

TABELA 1 - Média dos testes de emissão de gases atmosféricos.

\begin{tabular}{|c|c|c|}
\hline Gás & $610 \mathrm{rpm}^{\star}$ & $2500 \mathrm{rpm}^{\star \star}$ \\
\hline $\mathrm{O}_{2}$ & $0,10 \%$ & $0,00 \%$ \\
\hline $\mathrm{CO}_{2}$ & $11,60 \%$ & $11,70 \%$ \\
\hline NO & $0,00 \%$ & $0,00 \%$ \\
\hline $\mathrm{SO}_{2}$ & $0,00 \%$ & $0,00 \%$ \\
\hline $\mathrm{NO}_{\mathrm{x}}$ & $0,00 \%$ & $0,00 \%$ \\
\hline Temp. gás & $184,8^{\circ} \mathrm{C}$ & $422,8^{\circ} \mathrm{C}$ \\
\hline Temp. ar & $29,0^{\circ} \mathrm{C}$ & $29,0^{\circ} \mathrm{C}$ \\
\hline co & $0,02 \%$ & $0,03 \%$ \\
\hline $\mathrm{CO}_{\text {corrigido }}$ & $0,02 \%$ & $0,03 \%$ \\
\hline $\mathrm{HC}$ & $6 \mathrm{ppm}$ & 58 ppm \\
\hline$H_{\text {corrigido }}$ & $6,0 \mathrm{ppm}$ & $58,5 \mathrm{ppm}$ \\
\hline
\end{tabular}

${ }^{*}$ Marcha lenta
${ }^{* *}$ Marcha Alta

A Tabela 2 apresenta o comparativo dos valores médios obtidos nos testes com o ônibus e os limites propostos pela Resolução 418/2009, sendo que estes se apresentaram consideravelmente abaixo do permitido. 
TABELA 2 - Limites máximos de emissão de $\mathrm{CO}_{\text {corrigido e }} \mathrm{HC}_{\text {corrigido }}$

\begin{tabular}{rcc}
\hline Valor calculado & $\begin{array}{c}\text { Limites de emissão segundo a } \\
\text { CONAMA no 418/2009 }\end{array}$ & Resultado dos testes \\
\hline $\mathbf{C O}_{\text {corrigido }}$ & $1,0 \%$ & $0,025 \%$ \\
$\mathbf{H C}_{\text {corrigido }}$ & $500 \mathrm{ppm}$ & $58,5 \mathrm{ppm}$ \\
\hline
\end{tabular}

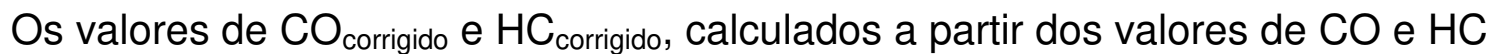
medidos são os únicos que contêm limites máximos estabelecidos em resolução. Os valores limites utilizados para esta comparação do biometano, foram os mesmos utilizados como limiares de Gás Natural Veicular de origem fóssil, pois as composições químicas são similares. Verificou-se que os poluentes $\mathrm{CO}$ e $\mathrm{HC}$ emitiram valores aproximadamente 40 e 10 vezes menores quando comparados com a CONAMA no 418/2009.

Os resultados destes testes se aproximaram do estudo feito por Kozerski e Hess (2006), que estimou com base em cálculos estequiométricos, os poluentes emitidos por ônibus e micro-ônibus utilizando diesel, biodiesel e gás natural, deduzindo-se que as emissões de gás natural são relativamente inferiores às dos demais combustíveis, equiparando-se aos achados desta pesquisa.

$\mathrm{Na}$ Tabela 3 são expostos os valores dos testes de poluição sonora, realizados conforme as NBR's 6067 e 9714, que prescrevem o método de ensaio para medição do ruído emitido nas proximidades do sistema de escapamento de veículos rodoviários automotores, na condição parado.

TABELA 3 - Média de nível de ruído emitido pelo veículo na condição estático

\begin{tabular}{ccccc}
\hline No de rotações & Teste 1 & Teste 2 & Teste 3 & Média \\
\hline 610 rpm $^{*}$ & 76,0 & 80,9 & 75,7 & 77,53 \\
2500 rpm ${ }^{\star *}$ & 96,0 & 95,2 & 95,8 & 95,67 \\
\hline${ }^{*}$ Marcha lenta & & &
\end{tabular}

Os resultados referentes aos níveis de ruídos apresentados na Tabela 3 , quando comparados com a Resolução 418/2009 (Tabela 4), atendem aos limites estabelecidos para o tipo de veículo em estudo, o qual se enquadra como veiculo de passageiros ou de uso misto com mais de nove lugares e PBT acima de $3.500 \mathrm{~kg}$.

TABELA 4 - Limites máximos de ruído emitidos por veículos na condição estática

\begin{tabular}{lc}
\hline Categoria & $\begin{array}{c}\text { Nível de } \\
\text { ruído (dB) }\end{array}$ \\
\hline $\begin{array}{l}\text { Veículo de passageiros até nove lugares e veículos de uso } \\
\text { misto derivado de automóvel. }\end{array}$ & 103 \\
$\begin{array}{l}\text { Veículo de passageiros com mais de nove lugares, veículo } \\
\text { de carga ou de tração, veículo de uso misto não derivado de }\end{array}$ & 103 \\
$\begin{array}{l}\text { automóvel e PBT até 3.500 kg. } \\
\text { Veículo de passageiros ou de uso misto com mais de } 9\end{array}$ & 98 \\
$\begin{array}{l}\text { lugares e PBT acima de 3.500kg. } \\
\text { Veículo de carga ou de tração com PBT acima de 3.500 kg. }\end{array}$ & 101 \\
\hline Fonte: Adaptado de CONAMA n 418/2009 &
\end{tabular}

Fonte: Adaptado de CONAMA n ${ }^{\circ} 418 / 2009$ 
Quanto às análises de consumo de combustível, foram percorridos $1.911 \mathrm{~km}$ entre os municípios de Montenegro, Lajeado, Triunfo e Portão, todos no Estado do Rio Grande do Sul, durante o período de um mês, com consumo de $897 \mathrm{~m}^{3}$ de biometano. Ou seja, em média o rendimento obtido foi de $2,13 \mathrm{~km} / \mathrm{m}^{3}$, representando resultado significativamente satisfatório considerando que o veículo testado se encontrava sem carga. Além disto, considerou-se que o Biometano tem as mesmas características do gás natural, estabelecidas na Lei 14.864/2016.

Em seus estudos Oliveira Filho et al. (2012), avaliaram o desempenho de duas frotas de ônibus no estado do Rio Grande do Norte utilizando três diferentes tipos de biodisel, mineral, B5 e B20. Constataram que houveram variações no desempenho entre os tipos de biodisel os quais apresentaram médias de 2,42;2,39 e 2,28 km/litro respectivamente. Desta forma, empiricamente comparando diretamente com os achados desta pesquisa a diferença média no desempenho é de aproximadamente $10 \%$.

\section{CONCLUSÕES}

No Brasil, após a aprovação de resolução ANP no 8 de 30/01/2015 e a Lei no 14.864/2016, percebe-se grande movimentação de investidores do setor buscando novos mercados para viabilizar o biometano como boa opção para substituição de outras fontes não renováveis ou renováveis de ciclo longo de combustível (BRASIL, 2015).

Considerando-se que os meios de transporte apresentam-se como um dos setores mais poluentes e causadores de impactos negativos ao meio ambiente, nossos achados reforçam o potencial da utilização do biometano como substituto interessante aos combustíveis fósseis, resultando em menores danos ao ambiente e menor risco à saúde pública, devido à baixa emissão de poluentes.

Notou-se que existe um pequeno número de pesquisas para servir como referências teóricas e metodológicas para avaliações e análises qualitativas do biometano. Neste trabalho, conforme descrito nos resultados, para a realização das comparações, utilizou-se legislações referentes ao GNV e estudos referentes a outros biocombustíveis.

Cabe ressaltar que estes foram testes pontuais realizados em um curto período de tempo, com todos os efeitos atmosféricos da região específica do Rio Grande do Sul, sendo assim, os resultados apresentados poderão sofrer alterações se replicados em outros momentos ou regiões. Para a obtenção de resultados mais aprofundados, sugere-se realizar avaliações em amostras com maior número de veículos, de diferentes modelos, frotas e regiões.

\section{AGRADECIMENTOS}

Os autores agradecem ao Centro Universitário Univates, à Companhia de Gás do Estado do Rio Grande do Sul (Sulgás), à Scania e ao Consórcio Verde Brasil pela confiança, contribuições e colaboração com o estudo.

\section{REFERÊNCIAS}

BILOTTA, P.; ROSS, B. Z. L. Estimativa de geração de energia e emissão evitada de gás de efeito estufa na recuperação de biogás produzido em estação de tratamento de esgotos. Eng Sanitaria Ambiental, v. 21, n. 21, p. 275-282, 2016. 
BRASIL. Ministério do Meio Ambiente, Resolução $n^{\circ}$ 418, de 25 de novembro de 2009. Conselho Nacional do Meio Ambiente - CONAMA. DOU $n^{0} 226$ de 26 de novembro de 2009, Brasil, 2009. Disponível em: <www.mma.gov.br>. Acesso em: 14 jul. 2016

BRASIL. Ministério do Meio Ambiente, $\mathbf{1}^{\circ}$ inventário nacional de emissões atmosféricas por veículos automotores rodoviários. Disponível em: <www.mma.gov.br>. Acesso em: 11 ago. 2016.

BRASIL. Agência Nacional do Petróleo Gás Natural e Biocombustíves, Resolução $n^{\circ}$ 8, de 30 de janeiro de 2015, DOU n 22 de 02 de fevereiro de 2015, Brasil, 2015. Disponível em: <www.anp.gov.br>. Acesso em: 20 ago. 2016

BRASIL. Agência Nacional do Petróleo Gás Natural e Biocombustíves, Resolução n 685, de 29 de junho de 2017, DO 30 de junho de 2017, Brasil, 2017. Disponível em: <www.anp.gov.br>. Acesso em: 05 nov. 2017

CÁCERES, C. X.; CÁCERES, R. E.; HEIN, D.; MOLINA, M. G.; PIA, J. M. Biogas production from grape pomace: Thermodynamic model of the process and dynamic model of the power generation system. International Journal of Hydrogen Energy, v. 37, n. 13, p. 10111-10117, 2012.

COIMBRA-ARAÚJO, C. H.; MARIANE, L.; BLEY, C.; PIRES, E.; SATO, M. et al. Brazilian case study for biogas energy: Production of electric power, heat and automotive energy in condominiums of agroenergy. Renewable and Sustainable Energy Reviews, v. 40, p. 826-839, 2014.

DRUMM, F. C.; GERHARDT, A. E.; FERNANDES, G. D.; CHAGAS, P.; SUCOLOTTI, M. S. et al. Poluição atmosférica proveniente da queima de combustíveis derivados do petróleo em veículos automotores. Reget/UFSM, v. 18, p. 66-78, 2014.

EBA - European Biogas Association, 2011. Biogas Production in Europe - Biogas Report 2011, Brussels.

EPE - Empresa de Pesquisa Energética. RenovaBio: regras de comercialização dos biocpmbustíveis, Rio de Janeiro, 2017.

FREITAS, C.; BREMNER, S. A.; GOUVEIA, N.; PEREIRA, L. A. A.; SALDIVA, P. H. N. Internações e óbitos e sua relação com a poluição atmosférica em São Paulo , 1993 a 1997. Revista de Saúde Pública, v. 38, n. 6, p. 751-757, 2004.

GONÇALVES, F. L. T.; CARVALHO, L. M. V.; CONDE, F. C.; LATORRE, M. R. D. O.; SALDIVA, P. H. N. et al. The effects of air pollution and meteorological parameters on respiratory morbidity during the summer in São Paulo City. Enviromental International, v. 31, p. 343-349, 2005.

JOHNSON, D.; HELTZEL, R. Methane emissions measurements of natural gas components using a utility terrain vehicle and portable methane quantification system. Atmospheric Environment, v. 144, p. 1-7, 2016. 
KARLSSON, T.; KONRAD, O.; LUMI, M.; CASARIL, C. E.; SCHMEIER, N. P. et al. Manual Básico de Biogás. 1. ed. Lajeado: Editora da Univates - Rio Grande do Sul, 2014.

KOZERSKI, G. R.; HESS, S. C. Estimativa dos poluentes emitidos pelos ônibus e microônibus de campo grande/ms, empregando como combustível diesel, biodiesel ou gás natural. . Engenharia Sanitaria e Ambiental, v. 11, p. 113-117, 2006.

LUMI, M.; KONRAD, O.; MARDER, M. Potencial de geração de biogás a partir da suplementação de óleo de babaçu em dejetos de animais Potential of biogas generation from babassu oil supplementation in livestock manure. Ciência e natura, v. 37 , n. 42 , p. 756-766, 2015.

OLIVEIRA FILHO, M. F.; SILVA, L. C. da; ABREU, M. A. de; GALVÃO FILHO A.S.M.; FARIAS, A. C. M. et al. Avaliação experimental da sensibilidade do biodiesel B5 em motores diesel utilizado em frotas de ônibus urbano e interurbano. Holos, v. 3, p. 3-14, 2012.

RIO GRANDE DO SUL. Lei 14.864, de 11 de maio de 2016. DOU $n^{\circ} 90$ de 12 de maio de 2016. Institui a Política Estadual do Biometano, o Programa Gaúcho de Incentivo à Geração e Utilização de Biometano - RS-GÁS - e dá outras providências.Porto Alegre, Brasil, 2016.

STARR, K.; GABARRELL, X.; VILLALBA, G.; TALENS, L.; LOMBARDI, L. et al. Life cycle assessment of biogas upgrading technologies. Waste Management, v. 32, n. 5, p. 991-999, 2012.

VINOTH KUMAR, K.; KASTURI BAI, R. Solar greenhouse assisted biogas plant in hilly region - A field study. Solar Energy, v. 82, n. 10, p. 911-917, 2008. 